\title{
Effect of Film Thickness on the Electrical Properties of AlN Films Prepared by Plasma-Enhanced Atomic Layer Deposition
}

\author{
Halit Altuntas, Cagla Ozgit-Akgun, Inci Donmez, and Necmi Biyikli
}

\begin{abstract}
In this paper, AIN thin films with two different thicknesses, i.e., 7 and $47 \mathrm{~nm}$, were deposited at $200{ }^{\circ} \mathrm{C}$ on p-type Si substrates by plasma-enhanced atomic layer deposition using trimethylaluminum and ammonia. To investigate the electrical characteristics of these AIN films, MIS capacitor structures were fabricated and characterized using current-voltage and high-frequency (1 MHz) capacitance-voltage measurements. The results showed that the current transport mechanism under accumulation mode is strongly dependent on the applied electric field and thickness of the AIN film. Possible conduction mechanisms were analyzed, and the basic electrical parameters were extracted and compared for AIN thin films with different thicknesses. Compared with 7-nm-thick film, a 47-nm-thick AIN film showed a lower effective charge density and threshold voltage along with a higher dielectric constant.
\end{abstract}

Index Terms-Aluminum nitride (AIN), current transport, Fowler-Nordheim (FN) tunneling, Frenkel-Poole (FP) emission, plasma-enhanced atomic layer deposition (PEALD), trap-assisted tunneling (TAT).

\section{INTRODUCTION}

O VER the last few decades, the interest for aluminum nitride (AIN) thin films has increased considerably because of the material's superior physical properties, including high electrical resistivity, good thermal and chemical stability, low toxicity, and high ultrasonic velocity. Moreover, AlN exhibits a wide and direct bandgap of $6.2 \mathrm{eV}$ along with a relatively high dielectric constant as well. These properties have made AlN an attractive candidate for various electrical and optoelectronic device applications [1]-[4]. Owing to its high dielectric constant, AlN has been proposed as a potential alternative material for silicon dioxide $\left(\mathrm{SiO}_{2}\right)$ forming

Manuscript received April 27, 2015; revised August 27, 2015; accepted August 28, 2015. Date of publication September 28, 2015; date of current version October 20, 2015. The work of C. Ozgit-Akgun, I. Donmez, and N. Biyikli was supported in part by the National Nanotechnology Research Center and in part by the State Planning Organization of Turkey through the National Nanotechnology Research Center Project. The work of N. Biyikli was supported by the Marie Curie International Reintegration Grant through the NEMSmart Project under Grant PIRG05-GA-2009-249196. The review of this paper was arranged by Editor G. Ghione.

H. Altuntas is with the Department of Physics, Cankiri Karatekin University, Cankiri 18100, Turkey (e-mail: altunhalit@gmail.com).

C. Ozgit-Akgun, I. Donmez, and N. Biyikli are with the National Nanotechnology Research Center, Bilkent University, Ankara 06800, Turkey (e-mail: caglaozgit@gmail.com; incidonmez@gmail.com; biyikli@unam.bilkent.edu.tr).

Color versions of one or more of the figures in this paper are available online at http://ieeexplore.ieee.org.

Digital Object Identifier 10.1109/TED.2015.2476597 the gate dielectric in MIS device structures. A variety of methods, including sputtering [5], pulsed laser deposition [6], ion beam deposition [7], molecular beam epitaxy (MBE) [8], metal-organic chemical vapor deposition (MOCVD) [9], plasma-enhanced chemical vapor deposition [10], and plasmaenhanced atomic layer deposition (PEALD) [11], have been utilized to deposit AlN thin films.

The ALD is a low-temperature thin-film deposition method in which a rapidly increasing interest is observed since the early 2000s due to its critical role in Si-based microelectronics. In this technique, the substrate surface is exposed to gaseous reactants (i.e., precursors) in an alternating cyclic manner separated by evacuation/purge periods. The ALD technique is inherently self-limiting; once a monomolecular layer of a precursor is chemisorbed on the surface, the gas-solid reaction stops, since the precursor molecules of the same kind do not react with each other unless they face thermal decomposition. The unique self-limiting growth mechanism results in a nearperfect thickness uniformity and ultimate conformality even on complex 3-D structures. When compared with alternative thin-film coating techniques, ALD stands out with the aforementioned critical superiorities enabling deposition at low temperatures and resulting in excellent thickness control at subnanometer scale producing uniform and conformal coatings [12]-[15]. Moreover, using the additional degree of freedom created by the plasma source in PEALD systems, in which the plasma source creates highly reactive radicals that contribute to the chemical reactions occurring at the substrate surface, it is possible to obtain a wider spectrum of materials at significantly lower temperatures.

In our previous studies, we reported on the self-limiting growth of polycrystalline AIN films at low temperatures by PEALD using trimethylaluminum (TMA) and ammonia $\left(\mathrm{NH}_{3}\right)$ as the aluminum and nitrogen precursors, respectively [16], [17]. In this paper, we have focused on the effect of film thickness on the electrical properties of AlN films prepared by PEALD at a substrate temperature of $200{ }^{\circ} \mathrm{C}$; conduction mechanisms for these AlN films were demonstrated, and the basic electrical parameters were determined and comparatively analyzed.

\section{EXPERIMENT}

MIS capacitor structures with AlN as the insulating layer were fabricated on $25-\mathrm{mm} \times 40$-mm p-type $\mathrm{Si}(100)$ 


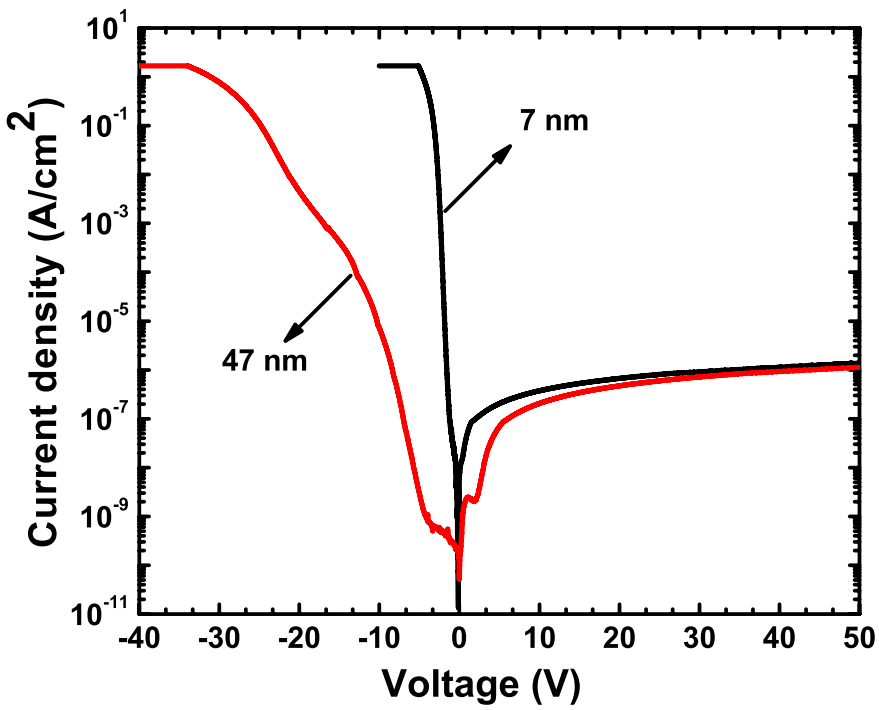

Fig. 1. $J-V$ characteristics of Al/AlN/p-Si MIS structure under forward and reverse bias plotted in semilog scale.

substrates $(\rho=20-40 \Omega \cdot \mathrm{cm})$ using cleanroom-based standard microfabrication process equipments and techniques. After cleaning the substrates, backside ohmic contacts were formed via thermal evaporation and subsequent rapid thermal annealing. The substrates with back ohmic contacts were then loaded into a Fiji F200-LL ALD reactor (Ultratech/CambridgeNanotech Inc.) immediately after they were dipped into dilute HF solution, rinsed with DI water and dried with $N_{2} ; 75$ and 575 cycles AlN were deposited on these substrates at $200{ }^{\circ} \mathrm{C}$; one PEALD cycle consisted of 0.1-s TMA pulse/10-s Ar purge/40 s, $50 \mathrm{sccm}$, and $300-\mathrm{W} \mathrm{NH}_{3}$ plasma/10-s Ar purge. The samples were solvent cleaned, and top contacts were formed via standard liftoff process where $\sim 80$-nm thick $\mathrm{Al}$ was thermal evaporated. $\mathrm{Al}$ and AlN layers were patterned simultaneously to obtain $250-\mu \mathrm{m} \times 250-\mu \mathrm{m}$ MIS devices. Room-temperature capacitance-voltage $(C-V)$ and current-voltage $(I-V)$ characteristics of the fabricated MIS capacitor structures were measured under dark. In addition, AlN films were characterized by X-ray photoelectron spectroscopy (XPS).

\section{RESULTS}

The current density $(J)$ curves at negative and positive bias voltages are shown in Fig. 1, which clearly indicates that both fabricated MIS structures show a rectifying behavior.

In order to investigate the current conduction mechanisms in 7- and 47-nm-thick AlN films, the part of forward-biased current density-voltage $(J-V)$ measurement (MIS capacitor structures were biased into the accumulation regime) results presented in Fig. 2(a) and (b), respectively, was analyzed. As shown in Fig. 2(a) and (b), current density strongly depends on the applied electric field, and both curves have three different regions with a distinct character.

To study carrier transport in dielectric films, several current conduction mechanisms have been suggested. For 7-nm-thick AlN films [Fig. 2(a)], at low electric fields (i.e., the region indicated by green arrows), the current density is proportional
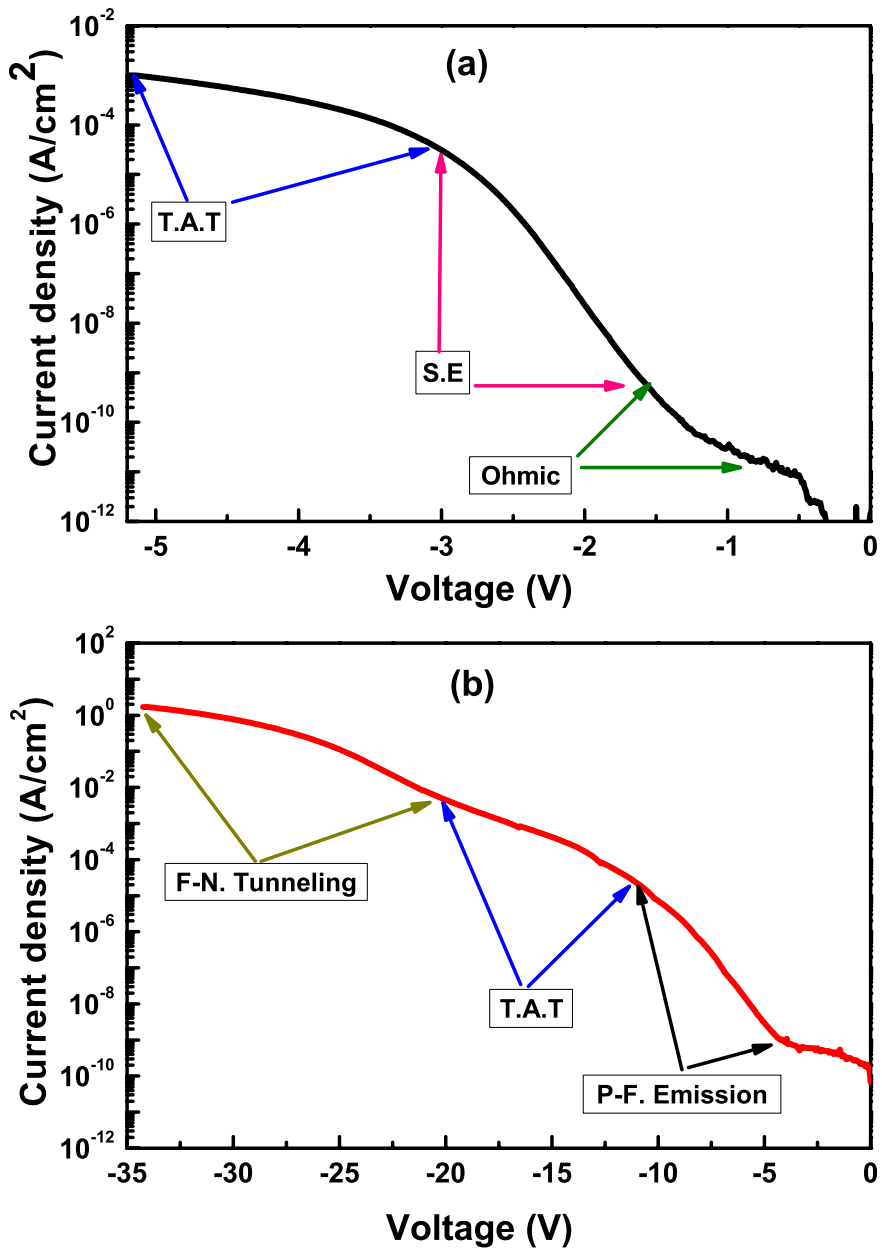

Fig. 2. Variation of current density with applied voltage for MIS capacitors with (a) 7- and (b) 47-nm-thick AlN films. Arrows: type of conduction mechanisms within certain voltage intervals.

to the electric field $(E)$, and the slope of the $\ln (J)$ versus ln $(E)$ plot (Fig. 3) is very close to 1 . This type of low field transport mechanism indicates typical ohmic conduction.

At medium electric fields [i.e., the region indicated by pink arrows in Fig. 2(a)], ln $(J)$ versus $E^{1 / 2}$ plot (Fig. 4) was fitted very well. Schottky emission (SE) mechanism is described as [18]

$$
J \propto A^{*} T^{2} \exp \left[\frac{-q\left(\phi_{B}-\sqrt{q E / 4 \pi \varepsilon_{r} \varepsilon_{o}}\right)}{k_{B} T}\right]
$$

where $J$ is the current density, $E$ is the electric field, $T$ is the temperature in kelvin, $A^{*}$ is the effective Richardson constant, $\phi_{B}$ is the barrier height, $\varepsilon_{r}$ is the dielectric constant of the dielectric films, $q$ is the electronic charge, and $k_{B}$ is the Boltzmann constant.

Using (1), $\varepsilon_{r}$ value was calculated as 1.2 from Fig. 4. This dielectric constant value is in reasonable agreement with the value (1.6) obtained from the $C-V$ measurement carried out at $1 \mathrm{MHz}$. Thus, we can state that for the 7-nm-thick AlN film, conduction mechanism is being governed by the SE in the range of medium electric fields.

At high electric fields [i.e., the region indicated by blue arrows in Fig. 2(a)], $\ln J$ versus $1 / E$ plot (Fig. 5) 


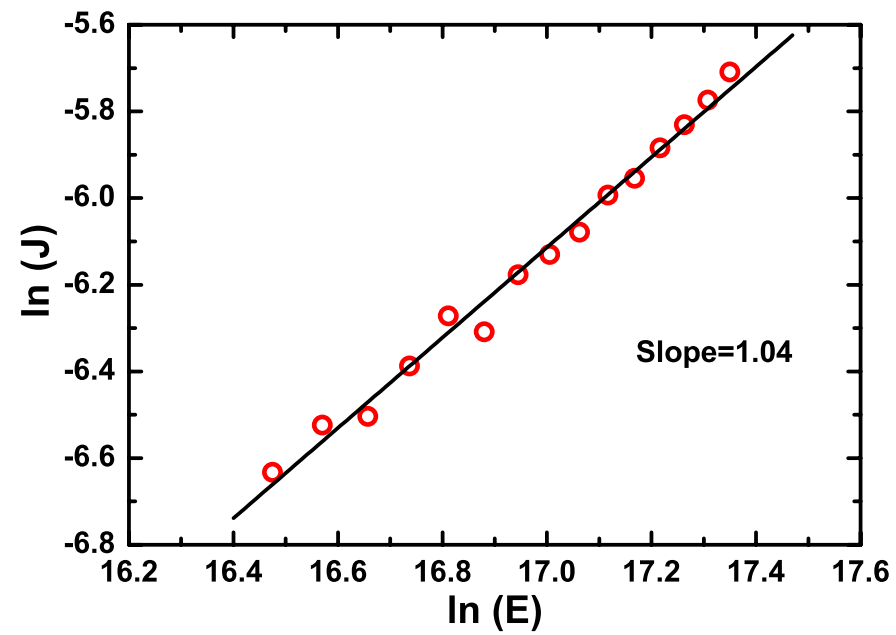

Fig. 3. Ln $(J)$ versus $\ln (E)$ plot of the MIS structure with 7-nm AlN films.

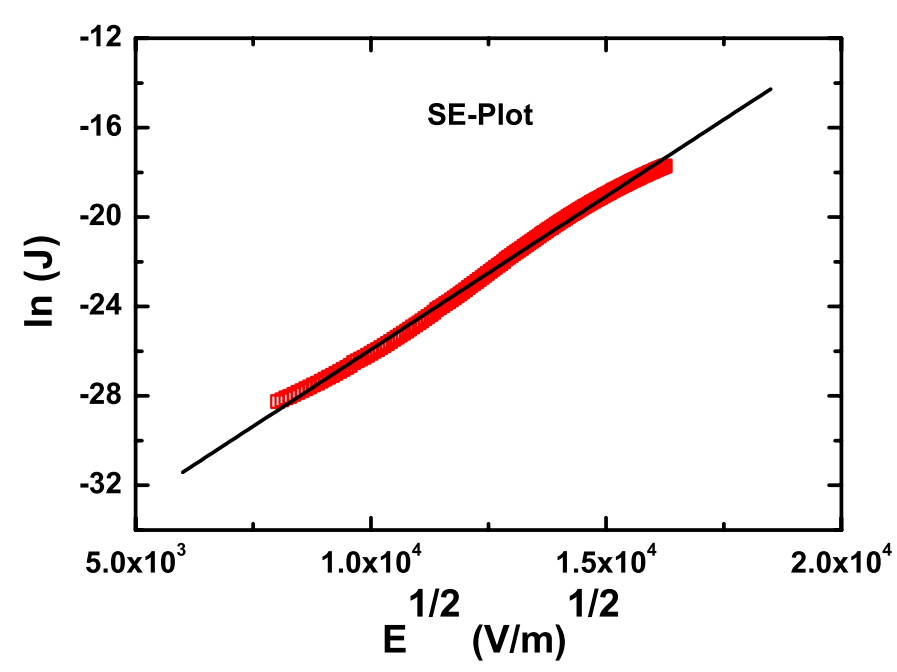

Fig. 4. SE plot of the MIS structure with 7-nm AlN films.

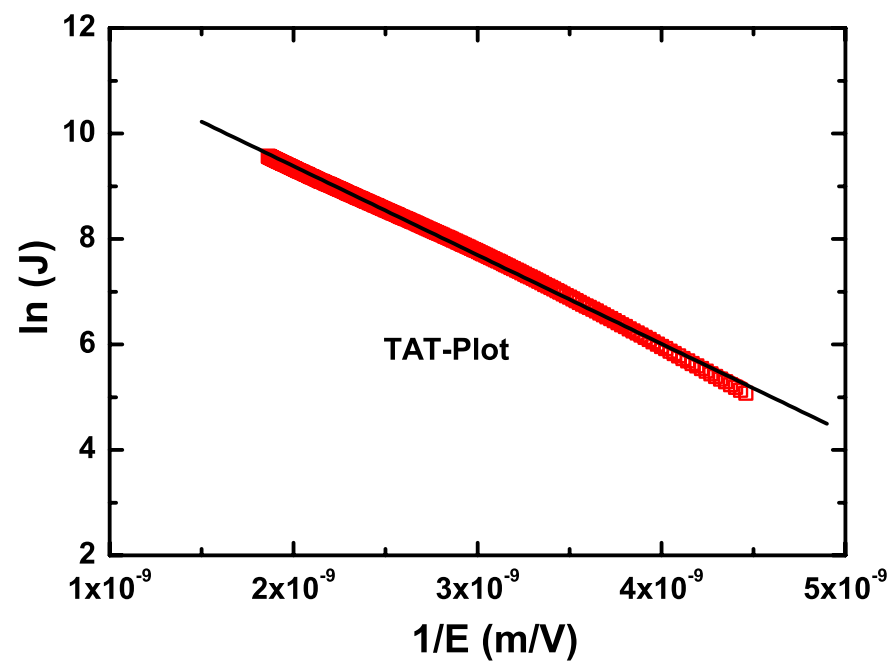

Fig. 5. TAT-plot of the MIS structure with 7-nm AlN films.

has a linear relationship, which indicates that the governing conduction mechanism is mainly due to trap-assisted tunneling (TAT).

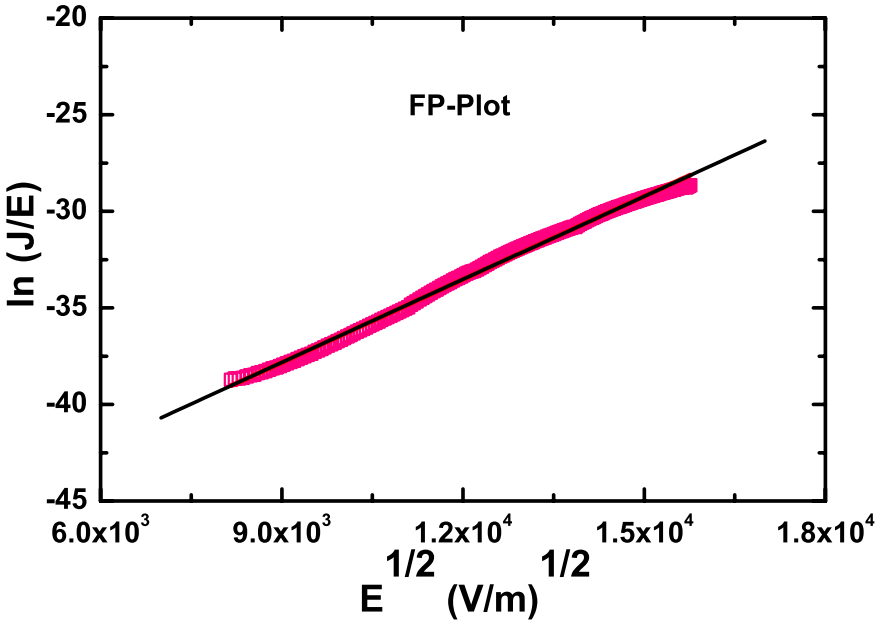

Fig. 6. FP plot of the MIS structure with 47-nm AlN films.

The current due to TAT mechanism is given by the following equation [18]:

$$
J_{\mathrm{TAT}} \propto \exp \left\{\frac{-8 \pi \sqrt{2 q m_{\mathrm{AIN}}}}{3 h E} \phi_{t}^{3 / 2}\right\}
$$

where $\phi_{t}$ is the trap energy level, which can be obtained from the $\ln J$ versus $1 / E$ plot. Using this equation, the trap energy level was obtained as $0.6 \mathrm{eV}$. The obtained trap energy level is equal to that predicted for a nitrogen vacancy of crystalline AlN in [19]. Also, Ligatchev et al. [20] have reported the values of $0.47-0.62$ and $0.73-0.80 \mathrm{eV}$ for defect states in AlN deposited on silicon. They identified those defect states as the levels of the nitrogen vacancies. Hence, the TAT mechanism is responsible for electron conduction through AlN, which is attributed to the existence of nitrogen vacancies in the film. In brief, ohmic conduction, SE, and TAT were found as the dominant transport mechanisms taking place at low, medium, and high electric field regions for MIS capacitors with 7-nm-thick AlN layers.

For 47-nm thick AlN films [Fig. 2(b)], the $\ln J / E$ versus $E^{1 / 2}$ plot (Fig. 6) was very well fitted at low electric fields (i.e., the region indicated by black arrows). Current density due to Frenkel-Poole (FP) emission can be given as [18]

$$
J \propto E \exp \left[\frac{-q\left(\phi_{t}-\sqrt{q E / \pi \varepsilon_{r} \varepsilon_{o}}\right)}{k_{B} T}\right]
$$

where $\phi_{t}$ is the trap energy barrier height in the AlN thin film and $\varepsilon_{r}$ is the dielectric constant of the dielectric film. From (3), the slope of Fig. 6 provides an estimate of the dielectric constant of AlN thin film. Thus, $\phi_{t}$ and $\varepsilon_{r}$ were calculated to be $1.4 \mathrm{eV}$ and 4.4 , respectively. The obtained dielectric constant is close to 6.2 , which was calculated from the high-frequency $C-V$ measurements. On the other hand, regarding to the trap level, Tansley and Egan [21] reported that the energy levels in AlN of about (1/4)Eg or $E_{C}-E=$ $1.4-1.8 \mathrm{eV}$ correspond to the $N_{\mathrm{Al}}$ antisite defects. In a previous study, we reported the bandgap of PEALD-grown AlN to be $5.8 \mathrm{eV}$ as determined by the optical spectrum transmission measurements [16]. A quarter of the obtained bandgap is equivalent to $1.45 \mathrm{eV}$, and this value coincides with the 


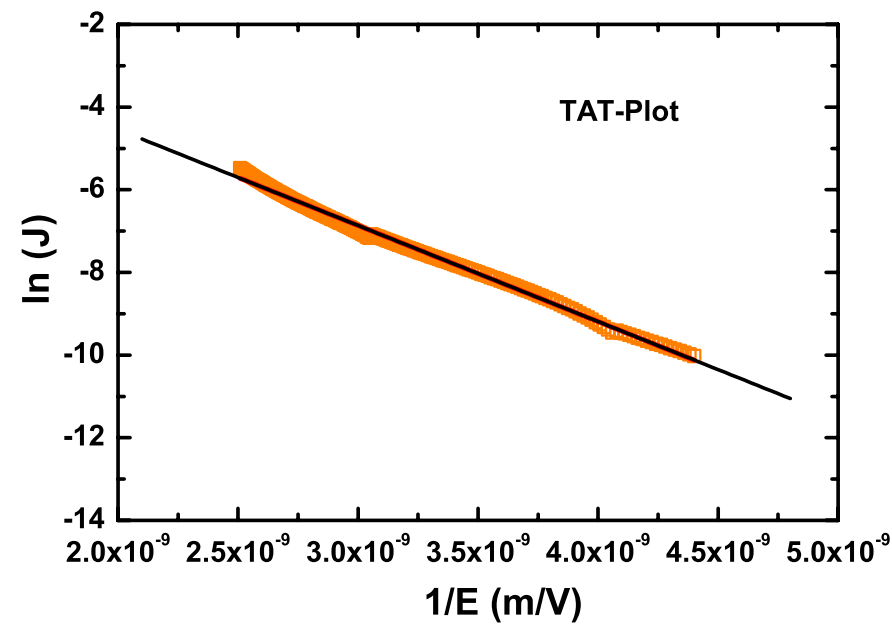

Fig. 7. TAT-plot of the MIS structure with 47-nm AlN films.

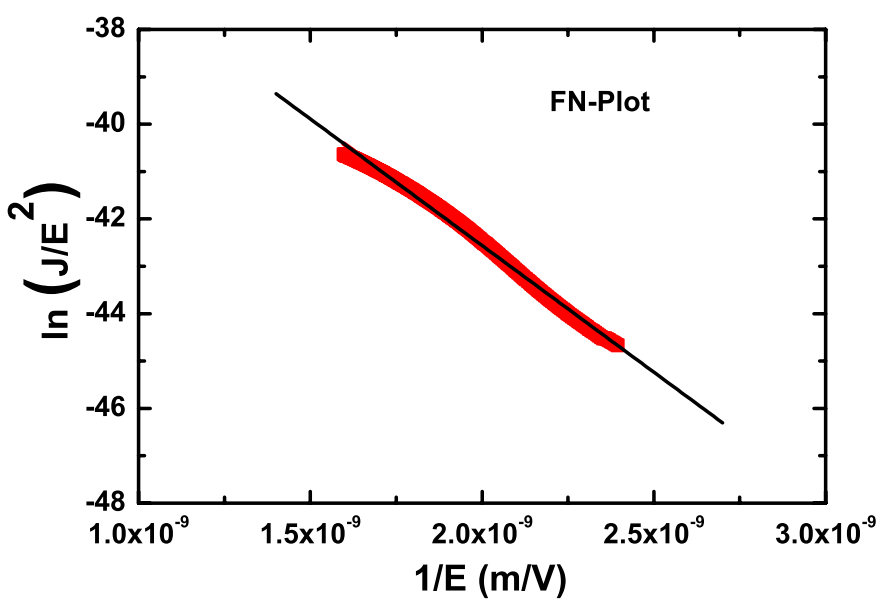

Fig. 8. FN-plot of the MIS structure with 47-nm AlN films.

trap level, which was calculated from the FP plot. Thus, the obtained trap level was attributed to the $N_{\mathrm{Al}}$ antisite defects in a 47 -nm-thick AlN film.

The $\ln J$ versus $1 / E$ plot (Fig. 7) was fitted very well at medium electric fields [i.e., the region indicated in Fig. 2(b) by blue arrows), which indicates that the conduction mechanism at these fields is due to TAT. By using (2), the trap level was calculated as $0.72 \mathrm{eV}$. Since this trap level is close to the range of $0.73-0.80 \mathrm{eV}$, it was attributed to the nitrogen vacancies.

Finally, at the high electric field range [i.e., the region indicated in Fig. 2(b) by green arrows), the $\ln \left(J / E^{2}\right)$ versus $1 / E$ plot (Fig. 8) has a linear relationship, which indicates that the dominating conduction mechanism is due to Fowler-Nordheim (FN) tunneling.

The FN tunneling current density is given by the following relation [18]:

$$
J_{\mathrm{FN}} \propto E^{2} \exp \left\{\frac{-8 \pi \sqrt{2 q m_{\mathrm{AlN}}}}{3 h E} \phi_{b}^{3 / 2}\right\}
$$

where $\phi_{b}$ is the barrier height at the emitting interface, and it can be calculated from the slope of $\ln \left(J / E^{2}\right)$ versus $1 / E$ plot. Using (4), $\phi_{b}$ was calculated as $1.17 \mathrm{eV}$.

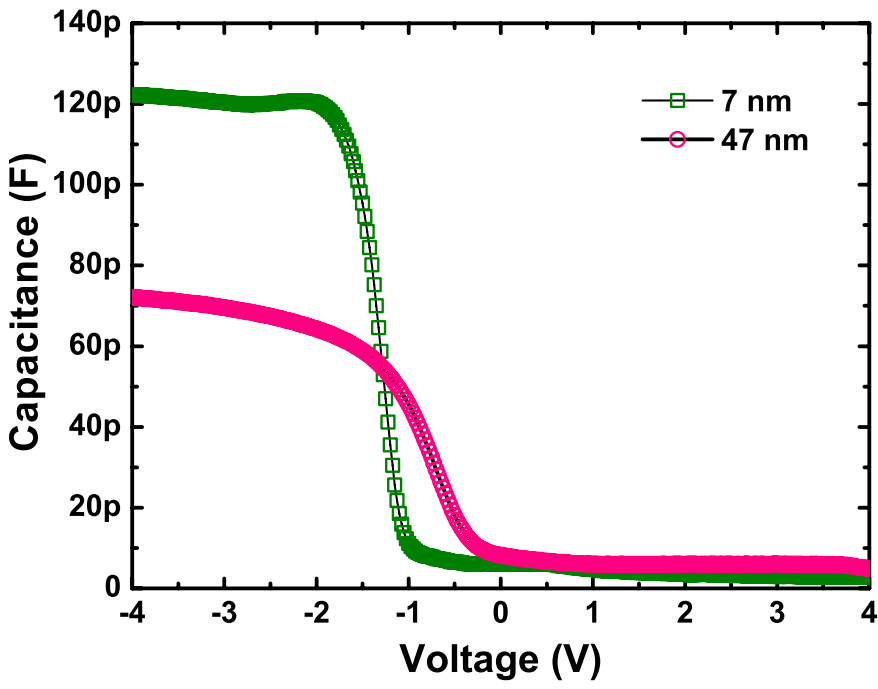

Fig. 9. Typical measured $C-V$ curves of $\mathrm{Al} / \mathrm{AlN} / p$-Si MIS structures with 7- and 47-nm-thick AlN insulating layers. Measurements were carried out at a frequency of $1 \mathrm{MHz}$.

This value is lower than the derived barrier height for the $\mathrm{Al} / \mathrm{AlN}$ interface. Therefore, an electron transferring from gate into the conduction band of the dielectric may be emitted from the defect levels due to FN tunneling [22]. Some researchers have presented results related to electrical conduction mechanisms of AlN thin films. Engelmark et al. [23] reported that the dominant mechanism responsible for conduction through the AlN film is due to FP emission. Breit et al. [24] observed ohmic conduction for low field and space charge limited current mechanism for high field. Hassine et al. [25] observed ohmic conduction for low field and FP emission for high field. Liufu and Kao [26] presented FN tunneling when the field is close to breakdown. Oikawa et al. [27] reported that possible mechanisms of the electrical conduction are FP emission or SE, in which some defects might act as carrier trapping centers. As known, different electrical conduction mechanisms can be observed for the same dielectric material depending on the applied electric field range, temperature, and dielectric layer thickness. Moreover, deposition technique utilized is another key factor, since deposition method and growth parameters are closely related to the occurrence of various defects and impurities. These lattice defects play an important role in carrier transport properties.

$C-V$ measurements were performed on the 7- and 47-nmthick AlN films (Fig. 9). From this result, one can conclude that the flat-band voltage exhibits a negative shift for both films, which indicates the existence of positive fixed dielectric charges trapped in the bulk AlN film/or in the dielectric/Si interface. Electrical and optical properties of the materials are influenced by the dielectric constant $\left(\varepsilon_{r}\right)$, and it can be obtained from the value of $C_{i}$ under strong accumulation mode by the following formula:

$$
C_{i}=\frac{\varepsilon_{r} \varepsilon_{o} A}{t_{d}}
$$

where $\varepsilon_{o}$ is the permittivity of vacuum and $t_{d}$ is the thickness of the dielectric layer. By the substitution of these parameters 
TABLE I

ATOMic Concentrations of Al, N, O, AND C AS Determined BY THE QUANTIFICATION OF XPS SURVEY SCANS

\begin{tabular}{|l|l|l|}
\hline & $\begin{array}{l}\text { 7 nm AlN } \\
\text { (Bulk film) }\end{array}$ & $\begin{array}{l}\text { 47 nm AlN } \\
\text { (Bulk film) }\end{array}$ \\
\hline Al (at.\%) & 40.73 & 49.46 \\
\hline N (at.\%) & 38.40 & 47.72 \\
\hline O (at.\%) & 20.21 & 2.27 \\
\hline C (at.\%) & 0.66 & 0.55 \\
\hline Al/N & 1.06 & 1.04 \\
\hline
\end{tabular}

into (5), the dielectric constants were found as 1.2 and 6.2 for the 7- and 47-nm-thick AlN films, respectively. The flatband voltages were calculated as -1.16 and $-0.82 \mathrm{~V}$ for 7- and 47-nm-thick AlN films, respectively. The estimated values of the effective charge densities $\left(N_{\text {eff }}\right)$ for 7 - and 47-nm-thick AlN films were found as $4.3 \times 10^{11}$ and $1.5 \times 10^{10} \mathrm{~cm}^{-2}$, respectively, using the flat-band voltage shift. With increasing film thickness, the amount of charge traps decreases, which reduces the flat-band voltage shift. Since $N_{\text {eff }}$ depends on the film thickness, fixed charges in the bulk AlN film are responsible for the shifting in $C-V$ curve.

Table I summarizes the XPS survey scan results. As can be seen from this table, the stoichiometry of 47-nm-thick AlN film is better than that of the 7-nm-thick films. Also, oxygen content in 47-nm AlN film is significantly lower (order of magnitude) than the 7-nm AlN films. Both observations might strong indicators that crystallinity increases with film thickness. Reduction in $N_{\text {eff }}$ with increasing film thickness might therefore be attributed to increased crystallinity.

Some studies related to effective charge densities of AlN films have been reported. For example, Stevens et al. [28] reported the order of $8 \times 10^{11} \mathrm{~cm}^{-2}$ for AlN films deposited by plasma-assisted MBE, Engelmark et al. [23] reported the order of $10^{12} \mathrm{~cm}^{-2}$ for AlN films deposited by reactive sputtering, Eom et al. [29] reported the order of $10^{12} \mathrm{~cm}^{-2}$ for AlN films deposited by sequential injection of TMA and $\mathrm{NH}_{3}$ under UV radiation, Olivera et al. [30] reported the order of $10^{12} \mathrm{~cm}^{-2}$ for AlN films deposited by dc magnetron sputtering, and Lee et al. [31] reported the order of $10^{13} \mathrm{~cm}^{-2}$ for AlN films deposited by RF magnetron sputtering. The values reported in this paper $\left(4.3 \times 10^{11}\right.$ and $\left.1.5 \times 10^{10} \mathrm{~cm}^{-2}\right)$ are better than in the literature for AlN thin films deposited using various methods and suggest that AlN thin films with low effective charge densities can be deposited successfully using low-temperature PEALD. The threshold voltage $\left(V_{\text {th }}\right)$ can be calculated for strong inversion of the MIS device (p-type substrate) according to [18]

$$
V_{\mathrm{th}}=V_{\mathrm{FB}}+2 \phi_{b}+\frac{\left(4 q \varepsilon_{\mathrm{Si}} \varepsilon_{o} N_{A}\left|\phi_{b}\right|\right)^{1 / 2}}{\varepsilon_{\mathrm{ox}} \varepsilon_{o} / t_{\mathrm{ox}}}
$$

where $\phi_{b}=\left(k_{B} T / q\right) \ln \left(N_{A} / n_{i}\right)$ and $n_{i}$ is the intrinsic carrier concentration of the silicon at room temperature (i.e., $1.45 \times 10^{10} \mathrm{~cm}^{-3}$ ). Substituting these parameters into (6) yields the $V_{\text {th }}$ values as -0.58 and $-0.19 \quad \mathrm{~V}$ for 7- and 47-nm-thick AlN thin films, respectively.

\section{CONCLUSION}

In summary, we have deposited polycrystalline wurtzite AlN films on p-type Si substrates at $200{ }^{\circ} \mathrm{C}$ by the PEALD technique utilizing an inductively coupled remote plasma source. To evaluate the effect of film thickness on the electrical conduction mechanisms and electrical parameters of AlN films, MIS device structures were fabricated along with subsequent $I-V$ and $C-V$ measurements. The 7- and 47-nm-thick AlN films showed different electrical transport mechanisms. Ohmic, SE, and TAT were found as the electrical conduction mechanisms in the 7-nm-thick AlN film. On the other hand, Poole-Frenkel emission, TAT, and FN tunneling were found as the electrical conduction mechanisms in the 47-nm-thick AlN film. The obtained trap levels were attributed to nitrogen vacancies and $N_{\mathrm{Al}}$ antisite defects within the bulk of AlN films. In our study, although the calculations for thin AlN films show that originating the traps of nitrogen vacancies play an active role in the conduction mechanism, it is readily oxidized in atmospheric environment, because it is thin and this situation may also affect the electrical properties of the film. Furthermore, the important electrical parameters of the capacitor structure, as well as the insulating layer, such as dielectric constant, flat-band voltage, effective charge density, and threshold voltage, were presented. The dielectric properties of 47-nm-thick AlN film were better compared with those of 7-nm-thick film. Our results show that AlN films with decent dielectric properties, which might have a great potential for various applications, can be deposited via PEALD using TMA and $\mathrm{NH}_{3}$ precursors at relatively low substrate temperatures.

\section{REFERENCES}

[1] A. Khan, K. Balakrishnan, and T. Katona, "Ultraviolet light-emitting diodes based on group three nitrides," Nature Photon., vol. 2, no. 2, pp. 77-84, Feb. 2008

[2] A. Khanna and D. G. Bhat, "Effects of deposition parameters on the structure of AlN coatings grown by reactive magnetron sputtering," J. Vac. Sci. Technol. A, vol. 25, no. 3, pp. 557-565, May/Jun. 2007.

[3] M. W. Jeong, S. W. Jeon, S. H. Lee, and Y. Kim, "Effective heat dissipation and geometric optimization in an LED module with aluminum nitride (AlN) insulation plate," Appl. Thermal Eng., vol. 76, no. 5, pp. 212-219, Feb. 2015.

[4] J. Zhang, Q. Zhang, H. Yang, H. Wu, J. Zhou, and L. Hu, "Bipolar resistive switching properties of AlN films deposited by plasma-enhanced atomic layer deposition," Appl. Surf. Sci., vol. 315, pp. 110-115, Oct. 2014

[5] M. A. Moreira, I. Doi, J. F. Souza, and J. A. Diniz, "Electrical characterization and morphological properties of AlN films prepared by dc reactive magnetron sputtering," Microelectron. Eng., vol. 88, no. 5, pp. 802-806, May 2011.

[6] Y. F. Lu, Z. M. Ren, T. C. Chong, B. A. Cheong, S. K. Chow, and J. P. Wang, "Ion-assisted pulsed laser deposition of aluminum nitride thin films," J. Appl. Phys., vol. 87, no. 3, pp. 1540-1542, Feb. 2000.

[7] C. Men and C. Lin, "A comparison of pulsed-laser-deposited and ion-beam-enhanced-deposited A1N thin films for SOI application," Mater. Sci. Eng., B, vol. 133, nos. 1-3, pp. 124-128, Aug. 2006.

[8] D. V. Nechaev et al., "Control of threading dislocation density at the initial growth stage of AlN on c-sapphire in plasma-assisted MBE," J. Crystal Growth, vol. 378, pp. 319-322, Sep. 2013.

[9] J. L. Dupuie and E. Gulari, "The low temperature catalyzed chemical vapor deposition and characterization of aluminum nitride thin films," J. Vac. Sci. Technol. A, vol. 10, no. 1, pp. 18-28, Jan./Feb. 1992. 
[10] T. Stauden, G. Eichhorn, V. Cimalla, J. Pezoldt, and G. Ecke, "The deposition of aluminum nitride on silicon by plasma-enhanced metal-organic chemical vapour deposition," Diamond Rel. Mater, vol. 5, no. 10, pp. 1210-1213, Aug. 1996.

[11] M. Bosund et al., "Properties of AlN grown by plasma enhanced atomic layer deposition," Appl. Surf. Sci., vol. 257, no. 17, pp. 7827-7830, Jun. 2011.

[12] S. Gieraltowska, L. Wachnicki, B. S. Witkowski, R. Mroczynski, P. Dluzewski, and M. Godlewski, "Characterization of dielectric layers grown at low temperature by atomic layer deposition," Thin Solid Films, vol. 577, pp. 97-102, Feb. 2015.

[13] M. Leskela, J. Niinisto, and M. Ritala, "Atomic layer deposition," Comph. Mater. Process., vol. 4, no. 5, pp. 101-123, Apr. 2014.

[14] M. Ritala, M. Leskelä, E. Nykänen, P. Soininen, and L. Niinistö, "Growth of titanium dioxide thin films by atomic layer epitaxy," Thin Solid Films, vol. 225, nos. 1-2, pp. 288-295, Mar. 1993.

[15] M. Vähä-Nissi et al., "Atomic and molecular layer deposition for surface modification," J. Solid State Chem., vol. 214, pp. 7-11, Jun. 2014.

[16] C. Ozgit, I. Donmez, M. Alevli, and N. Biyikli, "Self-limiting low-temperature growth of crystalline AlN thin films by plasma-enhanced atomic layer deposition," Thin Solid Films, vol. 520, no. 7, pp. 2750-2755, Jan. 2012.

[17] M. Alevli, C. Ozgit, I. Donmez, and N. Biyikli, "Structural properties of AlN films deposited by plasma-enhanced atomic layer deposition at different growth temperatures," Phys. Status Solidi A, vol. 209, no. 2, pp. 266-271, Feb. 2012.

[18] S. M. Sze and K. K. Ng, "Silicon MOS capacitors," in Physics of Semiconductor Devices, 3rd ed. New York, NY, USA: Wiley, 1981, pp. 209-229.

[19] D. W. Jenkins and J. D. Dow, "Electronic structures and doping of InN, $\operatorname{In}_{\mathrm{x}} \mathrm{Ga}_{1-\mathrm{x}} \mathrm{N}$, and $\mathrm{In}_{\mathrm{x}} \mathrm{Al}_{1-\mathrm{x}} \mathrm{N}$, " Phys. Rev. B, vol. 39, no. 5, pp. 3317-3329, Feb. 1989.

[20] V. Ligatchev, Rusli, and Z. Pan, "Density of defect states of aluminum nitride grown on silicon and silicon carbide substrates at room temperature," Appl. Phys. Lett., vol. 87, no. 24, pp. 242903-1-242903-3, Dec. 2005.

[21] T. L. Tansley and R. J. Egan, "Point-defect energies in the nitrides of aluminum, gallium, and indium," Phys. Rev. B, vol. 45, no. 19, pp. 10942-10950, May 1992.

[22] R. Perera, A. Ikeda, R. Hattori, and Y. Kuroki, "Trap assisted leakage current conduction in thin silicon oxynitride films grown by rapid thermal oxidation combined microwave excited plasma nitridation," Microelectron. Eng., vol. 65, no. 4, pp. 357-370, May 2003.

[23] F. Engelmark, J. Westlinder, G. F. Iriarte, I. V. Katardjiev, and J. Olsson, "Electrical characterization of AIN MIS and MIM structures," IEEE Trans. Electron Devices, vol. 50, no. 5, pp. 1214-1219, May 2003.

[24] F. Breit, T. Lebey, S. Agnel, and A. Toureille, "Investigations on DC conductivity of AlN," in Proc. Annu. Rep. Conf. Elect. Insul. Dielectr. Phenomena, Oct. 2001, pp. 71-74.

[25] N. B. Hassine et al., "Dielectrical properties of metal-insulator-metal aluminum nitride structures: Measurement and modeling," J. Appl. Phys., vol. 105, no. 4, pp. 044111-1-044111-10, Feb. 2009.

[26] D. Liufu and K. C. Kao, "Piezoelectric, dielectric, and interfacial properties of aluminum nitride films," J. Vac. Sci. Technol. A, vol. 16, no. 4, pp. 2360-2366, Jul./Aug. 1998.

[27] H. Oikawa et al., "Deposition and characterization of amorphous aluminum nitride thin films for a gate insulator," Thin Solid Films, vol. 574, pp. 110-114, Jan. 2015.

[28] K. S. Stevens, M. Kinniburgh, A. F. Schwartzman, A. Ohtani, and R. Beresford, "Demonstration of a silicon field-effect transistor using AlN as the gate dielectric," Appl. Phys. Lett., vol. 66, no. 23, pp. 3179-3182, Jun. 1995.

[29] D. Eom, S. Y. No, C. S. Hwang, and H. J. Kim, "Properties of aluminum nitride thin films deposited by an alternate injection of trimethylaluminum and ammonia under ultraviolet radiation," J. Electrochem. Soc., vol. 153, no. 4, pp. C229-C234, Feb. 2006.
[30] I. C. Oliveira, M. Massi, S. G. Santos, C. Otani, H. S. Maciel, and R. D. Mansano, "Dielectric characteristics of AlN films grown by d.c.-magnetron sputtering discharge," Diamond Rel. Mater., vol. 10, nos. 3-7, pp. 1317-1321, Mar./Jul. 2001.

[31] M.-S. Lee, S. Wu, S.-B. Jhong, K.-H. Chen, and K.-T. Liu, "Memory and electrical properties of (100)-oriented AlN thin films prepared by radio frequency magnetron sputtering," J. Nanomater, vol. 2014, pp. 250439-1-250439-6, Sep. 2014.

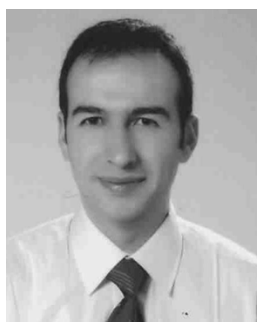

Halit Altuntas received the B.S., M.S., and Ph.D. degrees from the Department of Physics, Gazi University, Ankara, Turkey, in 2001, 2004, and 2009. respectively.

$\mathrm{He}$ is currently an Associate Professor with the School of Physics, Cankiri Karatekin University, Cankiri, Turkey, since 2011. His current research interests include electrical characterizations of thin films.

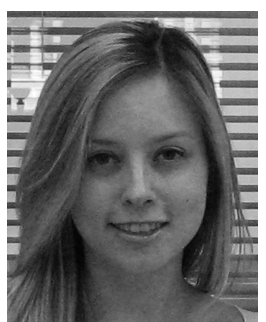

Cagla Ozgit-Akgun received the B.Sc. and M.Sc. degrees in metallurgical and materials engineering from Middle East Technical University, Ankara, Turkey, in 2006 and 2009, respectively, and the $\mathrm{Ph} . \mathrm{D}$. degree in materials science and nanotechnology from Bilkent University, Ankara, in 2014.

She is currently an Expert Engineer with the Microelectronics, Guidance and Electro-Optics Business Sector, ASELSAN Inc., Ankara.

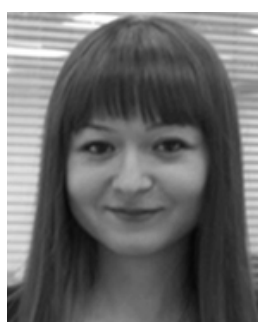

Inci Donmez received the B.S. degree in metallurgical and materials engineering from Middle East Technical University, Ankara, Turkey, and the M.S. degree in materials science and nanotechnology from Bilkent University, Ankara, in 2013. She is currently pursuing the Ph.D. degree with the Microelectronic Institute of Barcelona.

He has focused on the development of thermoelectric microgenerators based on silicon nanowires.

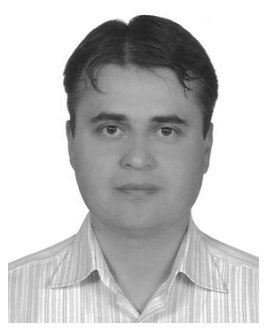

Necmi Biyikli received the B.S., M.S., and $\mathrm{Ph} . \mathrm{D}$. degrees from the Department of Electrical and Electronics Engineering, Bilkent University, Ankara, Turkey, in 1996, 1998, and 2004, respectively.

$\mathrm{He}$ is currently with the Institute of Materials Science and Nanotechnology, Bilkent University, leading the Functional Semiconductor Materials and Devices Research Group.

Dr. Biyikli contributed to more than 200 journal and conference publications and is a member of AVS and MRS 